\title{
Insulin secretion and insulin sensitivity in diabetic subgroups: studies in the prediabetic and diabetic state
}

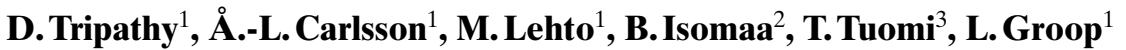 \\ ${ }^{1}$ Wallenberg Laboratory, Department of Endocrinology, Lund University, Sweden \\ 2 Jakobstad Hospital, Jakobstad, Finland \\ ${ }^{3}$ Department of Medicine, University Hospital, Helsinki, Finland
}

\section{Abstract}

Aims/hypothesis. To evaluate insulin sensitivity and insulin secretion in prediabetic and diabetic subjects with mutations in MODY1 $(H N F-4 \alpha)$ and MODY3 $(H N F-1 \alpha)$ genes, in subjects with GAD antibodies, latent autoimmune diabetes in adults and in subjects with the common form of Type II (non-insulin-dependent) diabetes mellitus.

Methods. Insulin secretion was measured as the incremental 30-min insulin (I30) and insulin glucose ratio (I:G30) during OGTT whereas insulin sensitivity was measured as the insulin sensitivity index during OGTT in 131 carriers of MODY mutations [NGT = 38 , IFG/IGT $=21$, diabetes mellitus $(\mathrm{DM})=72]$, in 293 subjects with GADA (NGT $=47, \mathrm{IFG} / \mathrm{IGT}=29$, $\mathrm{DM}=217)$ and in 2961 subjects with a family history of the common form of Type II diabetes but without MODY mutations or GADA $(\mathrm{NGT}=1360$, IFG/ $\mathrm{IGT}=857, \mathrm{DM}=744)$. A subgroup of the subjects underwent a euglycaemic clamp $(n=210)$ and intravenous glucose tolerance test $(n=337)$ for the estimation of insulin sensitivity and first-phase insulin secretion.

Results. Non-diabetic subjects with MODY mutations had pronounced impaired insulin secretion (I30, I:G30) compared with the two other groups ( $p=$ $0.005)$. Normal or non-diabetic glucose tolerance was maintained by enhanced insulin sensitivity compared with the other two groups $(p<0.05$ and $p<0.005)$. In contrast to patients with Type II diabetes and with adult latent autoimmune diabetes, MODY patients showed only a modest deterioration in insulin sensitivity at onset of diabetes. The 2 -h glucose values inversely correlated with insulin sensitivity in subjects with GADA $(r=-0.447, p<0.001)$ and subjects from Type II diabetic families $(r=-0.426, p<0.001)$, whereas no such relation was observed in subjects with MODY mutations $(r=0.151, p=\mathrm{NS})$. There were no statistically significant differences in insulin secretion or insulin sensitivity between subjects with GADA or subjects with a family history of Type II diabetes, either at the NGT or the IFG/IGTstage.

Conclusion/interpretation. Glucose-tolerant carriers of MODY mutations are characterised by a severe impairment in insulin secretion. Enhanced insulin sensitivity is the most likely explanation for the normal glucose tolerance. Whereas subjects with positive GADA or Type II diabetes have impaired insulin sensitivity with increasing glucose concentrations, MODY mutation carriers seem to be protected from the effect of glucose toxicity. [Diabetologia (2000) 43: 1476-1483]

Keywords LADA, MODY, Type II diabetes, IGT, insulin secretion, insulin sensitivity.
Received: 23 March 2000 and in revised form: 29 August 2000

Corresponding author: L. Groop, MD, Department of Endocrinology, Malmö University Hospital, Lund University, S-20502 Malmö, Sweden

Abbreviations: GADA, Glutamic acid decarboxylase antibodies; LADA, latent auto immune diabetes in adults; DM, diabetes mellitus; ISI, insulin sensitivity index; WHR, wait-to-hip ratio; M-value, insulin-stimulated glucose uptake during an euglycaemic clamp.
Diabetes is a far more heterogeneous disease than the current classification into Type I (insulin-dependent) diabetes mellitus and Type II (non-insulin-dependent) diabetes mellitus implies $[1,2,3]$. In the Scandinavian countries where approximately $3 \%$ of the population is known to have diabetes, $15 \%$ have Type I diabetes whereas $85 \%$ are considered to have Type II diabetes [4]. This is not entirely true, because about $10 \%$ of Caucasian patients with Type II 
diabetes have GADA or so-called latent auto immune diabetes in adults (LADA) [5, 6, 7]. In addition, among patients with early onset of familial diabetes younger than 40 years, about $15 \%$ carry mutations in MODY or mitochondrial genes [8].

Both insulin resistance and impaired insulin secretion contribute to the development of Type II diabetes [9]. Whereas insulin resistance and insulin secretory dysfunction are observed in first-degree relatives of patients with Type II diabetes [10, 11, 12], impaired insulin secretion usually determines the progression from IGT to diabetes [13]. These studies have considered, however, Type II diabetes as a rather homogenous disease.

To show the influence of disease-heterogeneity on the relative role of impaired insulin secretion and action in Type II diabetes, we assessed insulin secretion and insulin sensitivity during OGTTs in a large cohort of subjects with GADA or MODY mutations from the Botnia study. Insulin sensitivity and insulin secretion were also assessed in a subgroup of subjects by euglycaemic insulin clamp and intravenous glucose tolerance tests. The data show the importance of taking into account the heterogeneity of Type II diabetes when studying its pathogenesis.

\section{Subjects and methods}

The Botnia study was established in 1990 to identify genetic and metabolic factors contributing to the development of Type II diabetes in families from the western coast of Finland [14]. All patients with known Type II diabetes (response rate, $90 \%$ ) and their available family members (response rate about $75 \%$ ) from four primary care centres in the Botnia region were invited to participate. The study was subsequently extended to other parts of Finland and Sweden. Until now about 2500 subjects with diabetes and 7000 of their family members have been studied. We included all subjects who underwent an oral glucose tolerance test and had GAD antibody measurements. Subjects were labelled as MODY and latent autoimmune diabetes in adults (LADA) following screening for GAD antibody and the MODY mutations. Subjects were classified into different stages of glucose tolerance based upon their fasting and 2-h plasma glucose concentrations. Subjects with normal glucose tolerance (NGT) had fasting plasma glucose (FPG) of less than $6.1 \mathrm{mmol} / \mathrm{l}$ and 2-h glucose of less than $7.8 \mathrm{mmol} / \mathrm{l}$. Impaired glucose tolerance was defined as FPG 6.1 to $6.9 \mathrm{mmol} / \mathrm{l}$ or 2-h glucose value 7.8 to $11.1 \mathrm{mmol} / \mathrm{l}$ (IFG/IGT), or both. Subjects with diabetes (DM) had FPG of more than $7.0 \mathrm{mmol} / \mathrm{l}$ or 2 -h glucose of more than $11.1 \mathrm{mmol} / \mathrm{l}$, or both.

All subjects were screened for GADA. Subjects with GADA of more than 5 units were considered to have slowly progressing autoimmune diabetes, also referred to as LADA. Of 293 GADA positive subjects, 47 had NGT, 29 had IFG/ IGT and 217 had DM.

Screening for MODY mutations was carried out in 115 families with at least two members with onset of diabetes at a younger age than 40 years [8]. Mutations in the $H N F 1 \alpha$ gene (MODY3) were the most common mutations observed in 118 subjects, followed by mutations in the IPF-1 (MODY $4=18$ ), $H N F-1 \beta($ MODY $5=18), H N F-4 \alpha($ MODY $1=13)$ and glu- cokinase (MODY $2=6$ ) genes. We restricted our analysis to subjects with mutations in the $H N F-4 \alpha($ MODY1 = 13) and $H N F 1 \alpha($ MODY $3=118)$ genes. Of the subjects with a family history of Type II diabetes, but without GADA or MODY mutations, 1360 had NGT, 857 had IFG/IGT and 744 had DM.

Body weight and height were measured while subjects wore light clothing and no shoes. Waist circumference was measured with a soft tape midway between the lowest rib and the iliac crest while subjects were standing. Fat-free mass (FFM) was measured with infra red spectroscopy from the outer layer of the biceps in the dominant arm using a Futrex 5000 device (Futrex, Gaithersburg, Md., USA) [15]. Fat-free mass measured by this method correlates with the FFM obtained by bioelectrical impedance and the tritiated water techniques [15]. The coefficient of variation of repeated measurements by the same investigator was less than $1 \%$. Hip circumference was measured over the widest part of the gluteal region and waistto-hip ratio was calculated accordingly. Samples for the measurement of $\mathrm{HbA}_{1 \mathrm{c}}$, cholesterol, high density lipoprotein (HDL) cholesterol and triglycerides were drawn after an overnight fast. A standard (75-g) oral glucose tolerance test (OGTT) was done in all subjects with FPG of less than $11.1 \mathrm{mmol} / \mathrm{l}$ after overnight fasting. Blood samples for the measurement of plasma glucose and serum insulin were drawn at $-10,0,30,60$ and $120 \mathrm{~min}$.

Measures of insulin secretion. As indices of insulin secretion we used the early insulin response during OGTT (I 30) and the change in insulin/glucose during the first $30 \mathrm{~min}$ of the OGTT (I:G30) [16]. A subgroup of subjects underwent an intravenous glucose tolerance test (IVGTT, $n=337$ ). The number of subjects in different subgroups in whom IVGTT was carried out were: MODY, $n=17$ (NGT, 7; IFG/IGT, 4; DM, 6), LADA, $n=37$ (NGT, 19; IFG/IGT, 9; DM, 9) and Type II, $n=283$ (NGT, 141; IFG/IGT, 122; DM, 20), respectively. In brief, $0.3 \mathrm{~g} /$ $\mathrm{kg}$ body weight of a $50 \%$ glucose solution was given at time 0 . Blood samples for the measurement of insulin and blood glucose were obtained at $-10,0,2,4,6,8,10,20,40,50$, and $60 \mathrm{~min}$. The first-phase insulin response was calculated as the incremental insulin response during the first $10 \mathrm{~min}$ of the study.

Measures of insulin action. Insulin sensitivity was measured as the insulin sensitivity index (ISI) from the OGTT [17].

\section{0}

$\sqrt{[(\text { mean insulin } \times \text { mean glucose }) \times(\text { fasting glucose } \times \text { fasting insulin })]}$

Insulin action was also measured in a subgroup of subjects by a hyperinsulinaemic euglycaemic clamp. The number of subjects in different subgroups for whom euglycaemic clamps were done were, MODY, $n=11$ (IFG/IGT, 5; DM, 6), LADA, $n=40$ (NGT, 18; IFG/IGT, 10; DM, 12) and Type II, $n=159$ (NGT, 105; IFG/IGT, 40; DM, 14), respectively. After a priming dose of insulin, an infusion (infusion rate $45 \mathrm{mU} / \mathrm{m}^{2}$ ) of short-acting human insulin (Actrapid, Novo Nordisk, Denmark) was started and continued for $120 \mathrm{~min}$. Blood samples for the measurement of blood glucose were obtained at $5 \mathrm{~min}$ intervals throughout the clamp. A variable glucose infusion of $20 \%$ glucose was started to maintain blood glucose concentration constant at $5.5 \mathrm{mmol} / \mathrm{l}$ with a coefficient of variation $(\mathrm{CV})$ of $6 \%$. Insulin sensitivity was calculated from the glucose infusion rates during the last $60 \mathrm{~min}$ of the euglycaemic clamp and expressed as glucose uptake per lean body mass.

Assays. Plasma glucose during the clamp was measured with a glucose oxidation method, using a Beckman Glucose Analyzer 
Table 1. Clinical and metabolic characteristics of MODY mutation carriers, GADA positive subjects and relatives of Type II diabetic subjects with normal glucose tolerance

\begin{tabular}{|c|c|c|c|}
\hline & MODY & GADA & NGT \\
\hline$n=($ men $/$ women $)$ & $38(14 / 24)$ & $47(19 / 28)$ & $1360(605 / 755)$ \\
\hline Age $(\text { years })^{\mathrm{a}}$ & $28.5(12.1-41)$ & $50.5(34.7-56.3)$ & $43.7(34.2-55.7)$ \\
\hline $\operatorname{BMI}\left(\mathrm{kg} / \mathrm{m}^{2}\right)^{\mathrm{a}}$ & $20.8(19.2-23.2)$ & $25.3(22.4-27.5)$ & $24.7(22.7-27.5)$ \\
\hline WHR (men) & $0.90(0.05)$ & $0.94(0.05)$ & $0.94(0.09)$ \\
\hline WHR (women) & $0.81(0.05)$ & $0.81(0.05)$ & $0.82(0.08)$ \\
\hline Systolic blood pressure (mmHg) & $120(20)$ & $126(12)$ & $124(24)$ \\
\hline 2-h glucose $(\mathrm{mmol} / \mathrm{l})$ & $5.87(5.1-6.7)$ & $5.42(4.8-6.5)$ & $5.76(4.97-6.5)$ \\
\hline Fasting serum insulin (mU/l) & $4.96(3.5-5.9)$ & $6.39(4.83-9.6)$ & $6.23(4.6-8.8)$ \\
\hline Fasting serum C peptide $(\mathrm{nmol} / \mathrm{l})^{\mathrm{a}}$ & $0.31(0.21-0.45)$ & $0.38(0.30-0.66)$ & $0.43(0.29-0.58)$ \\
\hline Insulin area $(\mathrm{mU} / \mathrm{l})^{\mathrm{a}}$ & $1975(1216-3091)$ & $3985(2580-5461)$ & $3898(2596-5459)$ \\
\hline Cholesterol (mmol/l) & $4.76(4.45-5.29)$ & $5.32(4.65-6.28)$ & $5.32(4.61-6.08)$ \\
\hline Triglycerides (mmol/l) & $0.92(0.55-1.25)$ & $1.01(0.77-1.52)$ & $1.01(0.77-1.39)$ \\
\hline
\end{tabular}

Data are expressed as uncorrected values with Median (interquartile range). ${ }^{a} p<0.05$, MODY vs GADA positive subjects and relatives of Type II diabetic subjects

II (Beckman instruments, Fullerton, Calif., USA). Serum insulin concentrations were measured with specific radioimmunoassay (Pharmacia, Uppsala, Sweden), with an interassay CV of $5 \%$. Serum C-peptide concentrations were measured with radioimmunoassay (Linco Research, St. Charles, Mo., USA) with an interassay CV of $9 \%$. Non-esterified fatty acid (NEFA) was measured by an enzymatic colorimetric method (Wako Chemicals, Neuss, Germany). Total cholesterol and triglyceride concentrations in serum were measured on a Cobas Mira analyser (Hoffman La Roche, Basel, Switzerland).

GAD antibody screening. Measurement of GADA was done by a radioimmunoprecipitation method using $35 \mathrm{~S} / \mathrm{labelled} \mathrm{re-}$ combinant human GAD65 produced by in vitro transcription translation [5]. The results are expressed as relative units, RU (sample cpm-mean cpm of three negative control subjects)/ (cpm of a positive internal reference serum-mean cpm of three negative control subjects) $\times 100$. The cut-off limit for positivity was 5 RU for GADA which represent mean +3 SD of 296 Finnish healthy control subjects. In the combined Autoantibody Workshop [18], the specificity and sensitivity of the GADA assay were $99 \%$ and $75 \%$, respectively.

Screening for MODY mutations. Mutation screening of the $H N F-1 \alpha$ and $H N F-4 \alpha$ genes were carried out with single strand conformation polymorphism (SSCP) analysis and direct sequencing. The sequencing was done with a thermosequence dye terminator cycle sequencing premix kit (Amersham Life Sciences, Cleveland, Ohio, USA) according to manufacturers instructions using ABI 373 DNA sequencer (Applied Bio systems, Foster city, Calif., USA). Each specific fragment was sequenced with the original primers in both directions and analysed using a sequence software package (Gene codes corporation, Ann Arbor, Mich., USA). Details of the primer sequences and the mutations have been described previously [8]. Two mutations were found in the $H N F-4 \alpha$ (MODY 1) gene, a deletion of two adenines at codon 99 (K99fsdelAA) in exon 3 and an insertion of a valine between codons L328- L329 (V328-329ins) in exon 8. Mutations found in the HNF 1- $\alpha$ gene were, R131Q, R 272C, and Pro291fsinsC, L107I, S315fsinsA and G375fsdelG [8].
Statistical analysis. Data are presented as means \pm SD for normally distributed variables, as median (interquartile range) for non-normally distributed variables. The statistical analysis was carried out with Number Cruncher Statistical Systems (NCSS; Kaysville, Utah, USA) statistical software. The significance of difference between frequencies was tested using the Chisquared or Fisher's exact tests and significance of difference between group means with analysis of covariance (ANCOVA) using age and BMI as covariates. The incremental area under the OGTT curve was calculated with the trapezoidal rule. A $p$ value of less than 0.05 was considered statistically significant.

\section{Results}

Normal glucose tolerance. The MODY mutation carriers were younger $(p<0.005)$ and had a lower BMI $(p<0.05)$ than the two other groups (Table 1$)$. Their fasting C-peptide concentrations $(p<0.005)$ as well as insulin response following oral glucose were considerably lower than in GADA positive $(p<0.05)$ or NGT relatives of patients with Type II diabetes $(p<0.005)$. Insulin secretion measured as the I30 or I:G30 was also lower in MODY mutation carriers than NGT relatives of Type II diabetic patients $(p<0.0005)$ or GADA positive NGT subjects $(p<0.005)$ (Figs. 1, 2, 3). The first-phase insulin secretion during IVGTT was $50 \%$ lower in MODY mutation carriers than in the two other NGT groups $(p<0.005)$. Insulin sensitivity measured as ISI was better in normoglycaemic MODY mutation carriers than in GADA positive subjects $(10.3 \pm 0.8$ vs $7.4 \pm 0.6, p=0.004)$ and NGT Type II diabetic subjects $(10.3 \pm 0.8$ vs $7.3 \pm 0.1, p=0.0005)$. To account for the difference in insulin sensitivity between MODY subjects and Type II and GADA positive subjects, the I30 and I:G30 were adjusted for insulin sensitivity (ISI); insulin secretion adjusted for insulin 

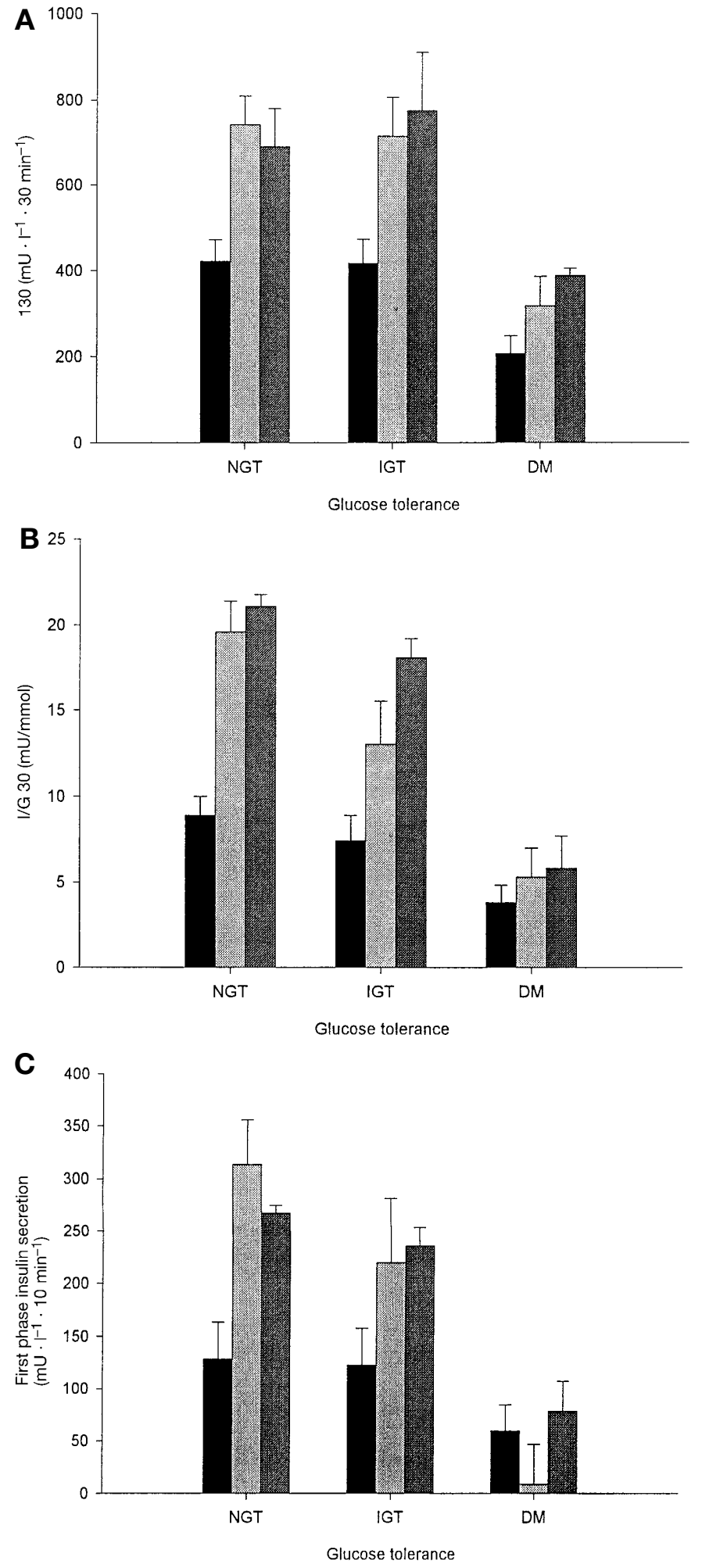

sensitivity was lower in MODY mutation carriers than in the other groups $(p<0.05)$. No difference in insulin secretion [I30, 742 (572 to 908 ) vs 702 (675 to $729), p=\mathrm{NS}$ ] or insulin sensitivity [ISI, 7.44 (6.0 to $8.8)$ vs 7.3 (7.08 to 7.48$), p=\mathrm{NS}$ ] was seen between subjects with GADA and relatives of Type II diabetic subjects with NGT (Figs. 1, 2).
Fig. 1. A Incremental insulin response (I30) during OGTT in

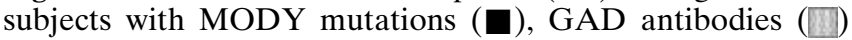
and subjects from families without GADA or MODY mutations ( $)$. A $p<0.005$ for the difference between MODY mutation carriers and GADA positive subjects or subjects from Type II diabetic families at all stages of glucose tolerance. Values are shown as means \pm SEM. B Ratio of incremental insulin and glucose (I/G30) during OGTT in subjects with MODY mutations ( $\mathbf{\square})$, subjects with GAD antibodies (D) and subjects from families without GADA or MODY mutations (). $p<0.005$ for difference between MODY mutation carriers and subjects from Type II diabetic families at all stages of glucose tolerance and MODY and GADA positive subjects with NGT and IGT. Values are shown as means \pm SEM. C First phase insulin response (IVGTT) in subjects with MODY mutations ( $\square$ ), subjects with GAD antibodies (D) and subjects from families without GADA or MODY mutations (). A $p<0.005$ for difference between MODY mutation carriers and GADA positive subjects and subjects from Type II diabetic families with NGT and $p<0.05$ between MODY mutation carriers and subjects from Type II diabetic families with IGT. Values are shown as means \pm SEM

Impaired glucose tolerance. Subjects with MODY had lower triglyceride $(p<0.05)$ and total cholesterol $(p<0.01)$ concentrations than IFG/IGT relatives of Type II diabetic subjects (Table 2). Both fasting Cpeptide concentrations and I30 and I:G30 were lower in MODY mutation carriers than in IFG/IGT subjects with GADA $(p<0.05)$ or subjects with a family history of Type II diabetes $(p<0.005)$. Subjects with MODY were more insulin sensitive at this stage as reflected by a higher ISI $(p<0.05)$ and a higher insulin mediated glucose uptake (M-value) during the euglycaemic clamp $(p<0.05)$ compared with GADA positive and IFG/IGTrelatives of Type II diabetic patients. No difference was observed between subjects with GADA and relatives of Type II diabetic subjects with IFG/IGT in insulin secretion [I30, 715 (510 to 920) vs 825 (785 to 865$), p=\mathrm{NS}$ ] and insulin sensitivity [ISI, 4.64 (3.37 to 5.9) vs 4.19 (4.0 to 4.37), $p=$ NS] (Table 2).

Overt diabetes. Subjects with MODY were younger and had an earlier onset and longer duration of diabetes than Type II and LADA subjects (Table 3 ). The fasting C-peptide concentrations $(p<0.0005)$ and I30 values $(p=0.005)$ were lower in MODY than Type II diabetic patients. The I:G30 ratio was lower in MODY subjects than in Type II diabetic $(p<0.005)$ and LADA $(p=0.04)$ patients. The MODY patients had better insulin sensitivity as shown by higher ISI $(p<0.005)$ and higher M-value $(p<0.05)$ compared with Type II or LADA subjects. In addition, MODY patients had lower total cholesterol $(p<0.05)$ and triglyceride $(p<0.005)$ concentrations and higher HDL cholesterol concentrations $(p=0.01)$ than subjects with Type II diabetes and LADA patients. Subjects with LADA had a lower waist-to-hip ratio (WHR) $(p<0.005)$ and systolic $(p<0.005)$ and diastolic 

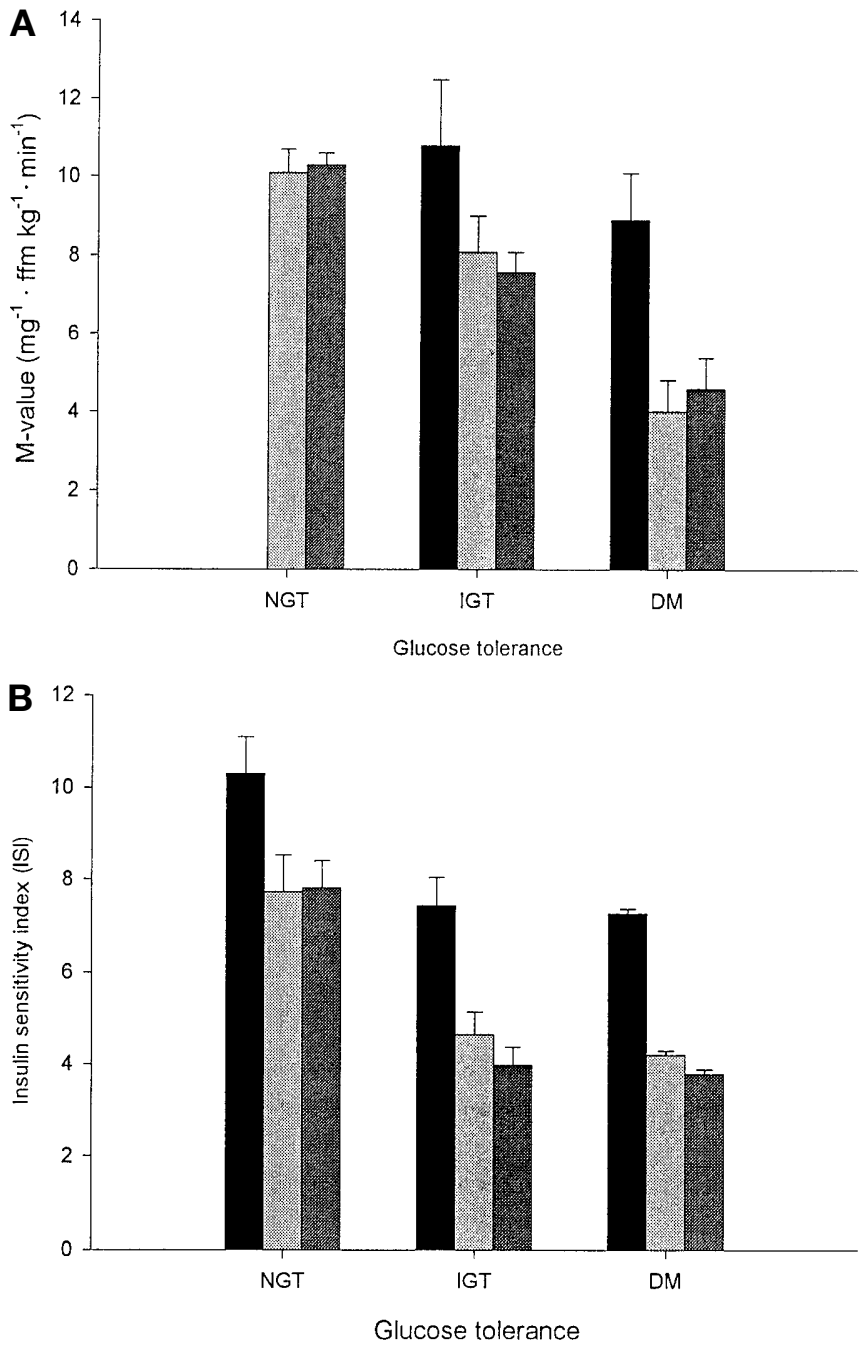

Fig. 2. A Insulin sensitivity index from OGTT in subjects with MODY mutations ( $\mathbf{\square})$, subjects with GAD antibodies (D) and subjects from families without GADA or MODY mutations ( ). A $p<0.005$ for difference between MODY and GADA positive and subjects from Type II diabetic families at all stages of glucose tolerance. Values are shown as means \pm SEM. B Insulin-mediated glucose disposal rates in relation to glucose tolerance in subjects with MODY mutations ( $\boldsymbol{\square})$, subjects with GAD antibodies (D) and subjects from families without GADA or MODY mutations ( $)$. A $p<0.05$ for difference between MODY mutation carriers and GADA positive subjects from Type II diabetic families with IGT and DM. Values are shown as means \pm SEM

$(p<0.005)$ blood pressure than Type II diabetic patients. Furthermore, they had lower triglyceride $(p=0.0007)$ and fasting C-peptide $(p<0.005)$ concentrations than Type II diabetic patients. No statistically significant difference in insulin sensitivity (ISI and Mvalues) was observed between the two groups (Table 3).

Correlation between insulin secretion (I30, I:G30) and insulin sensitivity (ISI) and glucose tolerance (2-h glu- cose). We also evaluated correlations (Pearson's) between the 2-h glucose concentration and measures of insulin secretion (I:G30, I30) and ISI in all subjects (NGT, IFG/IGT and DM) from the three groups. The 2-h glucose values showed a strong correlation with the I:G30 in subjects with GADA $(r=0.490$, $p<0.001)$, subjects from Type II diabetic families $(r=0.173, p<0.001)$ and subjects with MODY mutations $(r=0.495, p<0.001)$. The I30 also correlated with 2-h glucose in subjects with GADA $(r=0.447$, $p<0.001)$, subjects from Type II diabetic families $(r=0.300, p<0.001)$ and subjects with MODY mutation $(r=0.296, p=0.02)$. Whereas a higher 2-h glucose was associated with a lower ISI in subjects with GADA $(r=-0.447, p<0.001)$ and subjects from Type II diabetic families $(r=-0.426, p<0.001)$, no such relation was observed in subjects with MODY mutations $(r=0.151, p=\mathrm{NS})$.

\section{Discussion}

Although there is plenty of data showing impaired beta-cell function in diabetic MODY 3 mutation carriers [19], there is conflicting data on glucose tolerant people [19-23]. Other investigators [20] observed impaired insulin secretory response to stepped glucose infusions only at blood-glucose concentrations above $8 \mathrm{mmol} / \mathrm{l}$ and others [23] reported normal first-phase insulin response to intraveneous glucose in non-diabetic MODY 3 mutation carriers. In contrast, we observed considerably impaired insulin secretion in glucose-tolerant carriers of MODY 1 and MODY $3 \mathrm{mu}-$ tations regardless of whether insulin secretion was measured in response to oral (I30) or i.v. (FPIR) glucose or whether expressed relative to glucose concentrations (I:G30) or degree of insulin sensitivity $\left(\mathrm{I} 30_{\text {ISI }}\right)$. It is possible that different mutations in the $H N F 1 \alpha$ (or $H N F-4 \alpha$ ) gene might lead to different phenotypes. It is not likely though that this can explain the differences between the studies as we included subjects with six different mutations in the $H N F 1 \alpha$ gene (R131Q, R272C, Pro291fsinsC, L107I, S315fsinsA, G375fsdelG); the same (Pro291fsinsC) mutation was included in the other studies. Neither is it likely that inclusion of both MODY 1 and MODY 3 mutation carriers would have influenced the results; we as well as other investigators [20,21] have reported similar insulin secretory rates in subjects with MODY 1 and MODY 3 mutations. It should be noted that $H N F 4 \alpha$ is an upstream regulator of $H N F 1 \alpha$ and it is thus likely that mutations in either of them will lead to similar defects in insulin secretion [24].

The question arises of how the MODY mutation carriers can maintain normal glucose tolerance despite their severe impairment in beta-cell function. Enhanced insulin sensitivity could be one explanation. Alterations in insulin sensitivity as seen during 
Table 2. Clinical and metabolic characteristics of subjects with impaired glucose tolerance (IFG/IGT)

\begin{tabular}{|c|c|c|c|}
\hline & MODY & GADA & IFG/IGT \\
\hline$n=($ men/women $)$ & $21(8 / 13)$ & $29(10 / 19)$ & $857(412 / 445)$ \\
\hline Age (vears) $)^{\mathrm{a}}$ & $37.4 \pm 19$ & $57.6 \pm 17$ & $55.9 \pm 14$ \\
\hline $\operatorname{BMI}\left(\mathrm{kg} / \mathrm{m}^{2}\right)^{\mathrm{a}}$ & $22.4[20-26.4]$ & $26.8[24-31]$ & $26.8[25-30]$ \\
\hline WHR (men) & $0.92 \pm 0.07$ & $0.93 \pm 0.06$ & $0.96 \pm 0.06$ \\
\hline WHR (women) & $0.82 \pm 0.03$ & $0.84 \pm 0.04$ & $0.85 \pm 0.07$ \\
\hline Systolic blood pressure (mmHg) & $124[110-136]$ & $140[120-160]$ & $138[124-150]$ \\
\hline 2-h glucose $(\mathrm{mmol} / \mathrm{l})$ & $9.15[8.36-10.6]$ & $8.38[7.8-9.1]$ & $8.36[7.5-9.1]$ \\
\hline Fasting serum-Insulin (mU/l) & $7.95[4.6]$ & $9.1[6.1]$ & $9.32[6.9]$ \\
\hline Fasting serum-C peptide $(\mathrm{nmol} / \mathrm{l})^{a}$ & $0.31[0.21-0.45]$ & $0.66[0.42-0.71]$ & $0.56[0.39-0.76]$ \\
\hline Insulin area $(\mathrm{mU} / \mathrm{l})^{\mathrm{a}}$ & $2059[2631]$ & $4708[3890]$ & $5597[4985]$ \\
\hline Cholesterol $(\mathrm{mmol} / \mathrm{l})^{\mathrm{b}}$ & $4.42[3.85-5.46]$ & $6.37[4.90-6.92]$ & $5.79[5.17-6.52]$ \\
\hline Triglycerides $(\mathrm{mmol} / \mathrm{l})^{\mathrm{b}}$ & $0.86[0.68-1.12]$ & $1.19[0.96-2.27]$ & $1.62[1.01-1.96]$ \\
\hline
\end{tabular}

Data are expressed as uncorrected values with Mean \pm SD or Median (interquartile range). ${ }^{\mathrm{b}} p<0.05, \mathrm{MODY}$ vs IFG/IGT relatives of Type II subjects

${ }^{a} p<0.05$ MODY vs GADA positive subjects and IFG/IGT relatives of Type II diabetic subjects

Table 3. Clinical characteristics of subjects with diabetes

\begin{tabular}{|c|c|c|c|}
\hline & MODY & LADA & Type II \\
\hline$n=($ men/women $)$ & $72(39 / 33)$ & $217(92 / 125)$ & $744(432 / 412)$ \\
\hline Age $(\text { years })^{a}$ & $43.2 \pm 16$ & $56.4 \pm 18$ & $57 \pm 11$ \\
\hline Duration of diabetes (years) & $23.1 \pm 13$ & $11.5 \pm 9.6$ & $10.3 \pm 7.8$ \\
\hline Lean body mass (kg) & $53.5 \pm 11$ & $53.4 \pm 11$ & $55.1 \pm 11$ \\
\hline WHR $(\text { men })^{\mathrm{b}}$ & $0.95 \pm 0.07$ & $0.93 \pm 0.06$ & $0.96 \pm 0.07$ \\
\hline WHR (women) $)^{\mathrm{b}}$ & $0.88 \pm 0.07$ & $0.85 \pm 0.08$ & $0.88 \pm 0.06$ \\
\hline Fasting plasma-glucose $(\mathrm{mmol} / \mathrm{l})$ & $9.2(4.7)$ & $11.3(6.1)$ & $9.72(4.3)$ \\
\hline 2-h Glucose $(\mathrm{mmol} / \mathrm{l})$ & $15.8(6.6)$ & $18.0(10)$ & $15.8(7.7)$ \\
\hline Fasting serum-Insulin (mU/l) & $6.51(7.04)$ & $12.1(15.2)$ & $10.8(9.6)$ \\
\hline Fasting serum-C peptide $(\mathrm{nmol} / \mathrm{l})^{\mathrm{b}, \mathrm{c}}$ & $0.23(0.32)$ & $0.15(0.47)$ & $0.43(0.40)$ \\
\hline Insulin area $(\mathrm{mU} / \mathrm{l})^{\mathrm{a}}$ & $1453(1138)$ & $2049(3910)$ & $2535(2969)$ \\
\hline Cholesterol $(\mathrm{mmol} / \mathrm{l})^{\mathrm{a}}$ & $5.0(4.52-6.01)$ & $5.45(4.74-6.22)$ & $5.72(5.01-6.43)$ \\
\hline Triglycerides $(\mathrm{mmol} / \mathrm{l})$ & $1.20(0.87-1.7)$ & $1.11(0.88-1.7)$ & $1.54(1.08-2.23)$ \\
\hline
\end{tabular}

Data are expressed as uncorrected values with Means \pm SD or Median (interquartile range). ${ }^{\mathrm{b}} p<0.05$, LADA vs Type II DM

${ }^{\mathrm{a}} p<0.05$, MODY vs Type II DM and LADA, ${ }^{\mathrm{c}} p<0.05$, MODY vs Type II DM

infections and pregnancy could then trigger diabetes. In support of this, gestational diabetes is common among women with MODY mutations [8]. There was little or no deterioration of insulin sensitivity in MODY mutation carriers with IGT or diabetes, either when measured as ISI during OGTT or as insulin-stimulated glucose uptake during a euglycaemic clamp (M-value). These findings are in contrast to the findings in the GADA positive and subjects from families with the common form of Type II subjects, in whom a 40 to $50 \%$ decrease in insulin-stimulated glucose metabolism was seen in diabetic subjects compared with NGT subjects. The latter finding can partially be attributed to glucose toxicity [25], both the GADA positive and Type II diabetic subjects had increased glucose concentrations for several years. Patients with MODY on the contrary seem to be protected against the harmful effects of high-glucose concentrations on insulin sensitivity. There could be several reasons for this finding. A low renal threshold for glucose is a frequent corollary of MODY 3 mutations [26] and also a phenotypic characteristic of the $H N F-1 \alpha-/-$ mice [27]. Although not systematically evaluated in these patients, gross glucosuria was reported in several patients during the OGTT. Experimentally induced glucosuria with phlorizin in diabetic rats is able to reduce insulin resistance by preventing glucose toxicicity [28] and it is 
possible then that maintenance of normal or nearnormal insulin sensitivity in the MODY patients could be secondary to their low renal threshold for glucose and lack of glucose toxicity. It is, however, not known whether these transcription factors also directly influence the insulin signalling pathway in key organs like muscle, fat, liver and pancreas. Many genes harbour HNF response elements and patients with MODY 1 show very low triglyceride concentrations which can be ascribed to decreased expression of a key target of the $H N F-4 \alpha$ gene, i. e. apolipoprotein CIII, which acts as an inhibitor of lipoprotein lipase $[29,30]$.

There were no apparent differences in insulin sensitivity between GADA positive or subjects from families with Type II diabetes, either in the prediabetic or in the diabetic state. The fasting C-peptide concentrations and the I:G30 ratio tended to be, however, lower in the GADA positive subjects with NGT or IFG/IGT than the corresponding groups without MODY mutations or GADA. The causes leading to impaired beta-cell function are most likely different between the two groups; autoimmune destruction of the beta cells is most likely operative in LADA patients, whereas toxic effects of increased glucose [25], NEFA [31] and amylin [32] in addition to unknown factors are considered in the pathogenesis of beta-cell dysfunction in Type II diabetes. Despite these putative etiologic differences, the course of deterioration in insulin secretion seem to be similar and accompanied by a similar defect in insulin action in both groups. This could imply that the means to improve insulin sensitivity could have the same effect in both groups. It remains to be shown whether early insulin treatment is of benefit in LADA patients. Theoretically, beta-cell rest achieved by exogenous insulin could result in less exposure of beta-cell antigens to reactive T-cells [33].

Despite a similar degree of insulin resistance, the Type II diabetic subjects showed more features of the insulin resistance syndrome than the LADA patients, i.e. higher WHR, blood pressure and triglycerides. The dissociation between insulin resistance and features of the insulin resistance syndrome in LADA deserves some comments. Insulin resistance is an early and possibly inherited feature of first-degree relatives of patients with common Type II diabetes [34]. In contrast, normal sensitivity to insulin has been reported in offspring of LADA patients [35]. The insulin resistance observed in the LADA patients could thus represent secondary insulin resistance as a consequence of increased glucose or NEFA concentrations or both $[25,31]$. Whether insulin resistance in LADA and Type II diabetes really has different causes and whether the different risks of cardiovascular complications are related to intrinsic differences in the cause of insulin resistance or to different exposure times for insulin resistance is not yet known.
Of note, our data is restricted to subjects with a family history of diabetes. It is possible that subjects with NGT already showed some defects in insulin sensitivity or insulin secretion compared with subjects without a family history. For insulin sensitivity and insulin secretion this applies at least to NGT relatives from families with the common form of Type II diabetes $[11,14,36]$ whereas few data are available from GADA positive subjects with normal glucose tolerance. In addition, MODY mutation carriers with normal glucose tolerance have impaired insulin secretion compared with NGT subjects without a family history of diabetes [22].

It could be argued that the findings do not represent independent observations as several subjects came from the same pedigrees. This is not likely, as the results were virtually unchanged when only one subject from each nuclear family was included in the analysis.

The data clearly emphasize the heterogeneous nature of "Type II" diabetes and stress the importance of defining the underlying subtypes when studying the pathogenesis of the disease.

Acknowledgements. The skilful assistance of the Botnia Research group, is gratefully acknowledged. The study was financially supported by grants from the Sigrid Juselius Foundation, JDF-Wallenberg, Academy of Finland, Swedish Medical Research Council, Finnish Diabetic Research Foundation, Swedish Diabetic Research Foundation, EEC Paradigm and the Novo Nordisk Foundation.

\section{References}

1. Alberti KG, Zimmet P (1998) Definition, diagnosis and classification of diabetes mellitus and its implications. Part-1: Diagnosis and classification of diabetes mellitus, provisional report of a World Health Organisation consultation. Diabetic Med 15: 539-553

2. Groop LC, Tuomi T (1997) Non-insulin-dependent diabetes mellitus-a collision between thrifty genes and an affluent society. Ann Med 29: 37-53

3. American Diabetes Association Report of the expert committee on the diagnosis and classification of diabetes mellitus (1997). Diabetes Care 22 [Suppl 1]: S5-S19

4. Eriksson J, Forsen B, Haggblom M, Teppo AM, Groop L (1992) Clinical and metabolic characteristics of type 1 and type 2 diabetes: an epidemiological study from the Narpes community in western Finland. Diabet Med 9: 654-660

5. Tuomi T, Carlsson A, Li H, Isomaa B et al. (1999) Clinical and genetic characteristics of type 2 diabetes with and without GAD antibodies. Diabetes 48: 150-157

6. Zimmet PZ, Tuomi T, Mackay IR et al. (1994) Latent autoimmune diabetes mellitus in adults (LADA): the role of antibodies to glutamic acid decarboxylase in diagnosis and prediction of insulin dependency. Diabet Med 11: 299-303

7. Schranz DB, Bekris L, Landin-Olsson M et al. (2000) Newly diagnosed latent autoimmune diabetes in adults (LADA) is associated with low level glutamate decarboxylase (GAD65) and IA-2 autoantibodies. Diabetes Incidence Study in Sweden. Horm Metab Res 32: 133-138 
8. Lehto M, Wipemo C, Ivarsson SA et al. (1999) High frequency of mutations in MODY and mitochondrial genes in Scandinavian patients with familial early-onset diabetes. Diabetologia 42: 1131-1137

9. Beck-Nielsen H, Groop LC (1994) Metabolic and genetic characterization of prediabetic states. Sequence of events leading to non-insulin-dependent diabetes mellitus. J Clin Invest 94: 1714-1721

10. Eriksson J, Franssila-Kallunki A et al. (1989) Early metabolic defects in persons at increased risk for non-insulin-dependent diabetes mellitus. N Engl J Med 321: 337-343

11. Lillioja S, Mott DM, Spraul M, Ferraro R, Foley JE (1993) Insulin resistance and insulin secretory dysfunction as precursors of non insulin dependent diabetes mellitus. Prospective studies in PIMA Indians. N Eng J Med 329: 1988-1992

12. Pimenta W, Korytkowski M, Mitrakou A et al. (1995) Pancreatic beta-cell dysfunction as the primary genetic lesion in NIDDM. Evidence from studies in normal glucose-tolerant individuals with a first-degree NIDDM relative. JAMA 273: $1855-1861$

13. Weyer C, Bogardus C, Mott DM, Pratley RE (1999) The natural history of insulin secretory dysfunction and insulin resistance in the pathogenesis of type 2 diabetes mellitus. J Clin Invest 104: 787-794

14. Groop L, Forsblom C, Lehtovirta M et al. (1996) Metabolic consequences of a family history of NIDDM (The Botnia Study). Diabetes 45: 1585-1593

15. Franssila-Kallunki A (1992) Comparison of near-infrared spectroscopy, bioelectrical impendance and tritiated water technique for measurement of fat-free mass in human. Scand J Clin Lab Invest 52: 879-885

16. Phillips DI, Clark PM, Hales CN, Osmond C (1994) Understanding oral glucose tolerance: comparison of glucose or insulin measurements during the oral glucose tolerance test with specific measurements of insulin resistance and insulin secretion. Diabet Med 11: 286-292

17. Matsuda M, DeFronzo RA (1999) Insulin sensitivity indices obtained from oral glucose tolerance testing: comparison with the euglycemic insulin clamp. Diabetes Care 22: 1462-1470

18. Verge CF, Stenger D, Bonifacio E et al. (1998) Combined use of autoantibodies (IA-2 autoantibody, GAD autoantibody, insulin autoantibody, cytoplasmic islet cell antibodies) in type 1 diabetes: Combinatorial Islet Autoantibody Workshop. Diabetes. 47: 1857-1866

19. Lehto M, Tuomi T, Mahtani MM, Widen E et al. (1997) Characterization of the MODY3 phenotype. Early-onset diabetes caused by an insulin secretion defect. J Clin Invest 99: 582-591

20. Byrne MM, Sturis J, Menzel S et al. (1996) Altered insulin secretory responses to glucose in diabetic and nondiabetic subjects with mutations in the diabetes susceptibility gene MODY3 on chromosome 12. Diabetes 45: 1503-1510

21. Byrne MM, Sturis J, Fajans SS et al. (1995) Altered insulin secretory responses to glucose in subjects with a mutation in the MODY1 gene on chromosome 20. Diabetes 44: 699-704
22. Herman WH, Fajans SS, Smith MJ, Polonsky KS, Bell GI, Halter JB (1997) Diminished insulin and glucagon secretory responses to arginine in nondiabetic subjects with a mutation in the hepatocyte nuclear factor-4alpha (MODY1) gene. Diabetes 46: 1749-1754

23. Vaxillaire M, Pueyo ME, Clement K et al. (1999) Insulin secretion and insulin sensitivity in diabetic and non-diabetic subjects with hepatic nuclear factor-1alpha (maturity-onset diabetes of the young-3) mutations. Eur J Endocrinol 141: 609-618

24. Groop L, Lehto M (1999) Molecular and physiological basis for maturity onset diabetes of youth. Curr Opin Endocrinol Diabetes 6: 157-162

25. Yki, Jarvinen H (1990) Acute and chronic effects of hyperglycemia on glucose metabolism. Diabetologia 33: 579-585

26. Menzel R, Kaisaki PJ, Rjasanowski I, Heinke P, Kerner W, Menzel S (1998) A low renal threshold for glucose in diabetic patients with a mutation in the hepatocyte nuclear factor-1alpha (HNF-1alpha) gene. Diabet Med 15: 816-820

27. Pontoglio M, Barra J, Hadchouel M et al. (1996) Hepatocyte nuclear factor 1 inactivation results in hepatic dysfunction, phenylketonuria, and renal Fanconi syndrome. Cell 84: 575-585

28. Rossetti L, Smith D, Shulman GI, Papachristou D, De Fronzo RA (1987) Correction of hyperglycemia with phlorizin normalizes tissue sensitivity to insulin in diabetic rats. J Clin Invest 79: 1510-1515

29. Lehto M, Bitzen PO, Isomaa B et al. (1999) Mutation in the HNF-4alpha gene affects insulin secretion and triglyceride metabolism. Diabetes 48: 423-425

30. Navas MA, Munoz-Elias EJ, Kim J, Shih D, Stoffel M (1999) Functional characterization of the MODY1 gene mutations HNF4(R127W), HNF4(V255M), and HNF4(E276Q). Diabetes 48: 1459-1465

31. Ebeling P, Koivisto VA (1994) Non esterified fatty acids regulate lipid and glucose oxidation and glycogen synthesis in man. Diabetologia 37: 202-209

32. Kahn SE, Andrikopoulos S, Verchere CB (1999) Islet amyloid a long-recognized but underappreciated pathological feature of type 2 diabetes. Diabetes 48: 241-253

33. Vlahos WD, Seemayer TA, Yale J-F (1991) Diabetes prevention in $\mathrm{BB}$ rats by prophylactic insulin treatment. Metabolism 40: 825-829

34. Vauhkonen I, Niskanen L, Vanninen E, Kainulainen S, Uusitupa M, Laakso M (1998) Defects in insulin secretion and insulin action in non-insulin-dependent diabetes mellitus are inherited. Metabolic studies on offspring of diabetic probands. J Clin Invest 101: 86-96

35. Vauhkonen I, Niskanen L, Knip M et al. (2000) Impaired insulin secretion in non-diabetic offspring of probands with latent autoimmune diabetes mellitus in adults. Diabetologia 43: 69-78

36. Van Haeften TW, Dubbeldam S, Zonderland ML, Erkelens DW (1998) Insulin secretion in normal glucose tolerant relatives of type 2 diabetic subjects. Assessments using hyperglycemic glucose clamps and oral glucose tolerance tests. Diabetes Care 21: 278-282 